\title{
Erratum
}

\section{The ecology of Lake Nakuru (Kenya)}

\author{
V. Production and consumption of consumer organisms \\ E. Vareschi and J. Jacobs \\ Oecologia (Berlin) (1984) 61:83-98
}

On page 92 , right column, a calculation error occured. Line $21 \mathrm{ff}$. should read:

$50 \mu$ of Spirulina correspond roughly to $8 \cdot 10^{-7}$ cal. This means that $B$. plicatilis could not get its daily ration of energy from Spirulina even if a high assimilation efficiency is assumed. 